\title{
Energy Management Scheme for Wireless Powered D2D Users With NOMA Underlaying Full Duplex UAV
}

\author{
Ishan Budhiraja \\ Thapar Institute of Engineering \\ and Technology \\ Patiala, Punjab, India \\ ishanbudhiraja@gmail.com \\ Sudhanshu Tyagi \\ Thapar Institute of Engineering \\ and Technology \\ Patiala, Punjab, India \\ s.tyagi@thapar.edu
}

\author{
Neeraj Kumar \\ Thapar Institute of Engineering \\ and Technology \\ Patiala, Punjab, India \\ neeraj.kumar@thapar.edu \\ Sudeep Tanwar \\ Nirma University \\ Ahmedabad, Gujarat, India \\ sudeep.tanwar@nirmauni.ac.in
}

\author{
Mamoun Alazab \\ Charles Darwin University \\ Casuarina, Australia \\ mamoun.alazab@cdu.edu.au \\ Gaurav Srivastava \\ Brandon University \\ Brandon, Manitoba, Canada \\ srivastavag@brandonu.ca
}

\begin{abstract}
Device-to-Device (D2D) communications underlaying Unmanned aerial vehicle (UAV) with its mobility extend the coverage and improve the data rate. In this paper, we propose an energy management scheme for wireless powered D2D users with NOMA underlaying full-duplex (FD) UAV. Here, the cellular transmitters (CTs) and D2D transmitters (DDTs) first harvest energy from the radio frequency (RF) signals of the UAV. Then, the CT communicates with the cellular receivers (CRs) using the FD-UAV as a relay. On the other hand, DDT communicates with its two D2D receivers (DDRs) using the NOMA. We formulate the problem as a mixed-integer non-linear programming (MINLP) form and then divide it into two sub-problems. In the first sub-problem, an optimal value of time allocation for energy harvesting $(\mathrm{EH})$ for DMG is estimated, whereas, in the second subproblem, the power of DDT in each DMG is optimized using the variable changing technique. Finally, the joint time allocation and power control scheme is proposed to achieve the maximum energy-efficiency (EE). Numerical results demonstrated that the proposed scheme achieves better results as compared to the existing conventional NOMA and orthogonal multiple access (OMA) schemes.
\end{abstract}

\section{CCS CONCEPTS}

- Mathematics of computing $\rightarrow$ Coding theory; • Computer systems organization $\rightarrow$ Peer-to-peer architectures;

Permission to make digital or hard copies of all or part of this work for personal or classroom use is granted without fee provided that copies are not made or distributed for profit or commercial advantage and that copies bear this notice and the full citation on the first page. Copyrights for components of this work owned by others than ACM must be honored. Abstracting with credit is permitted. To copy otherwise, or republish, to post on servers or to redistribute to lists, requires prior specific permission a nd/or a fee. R equest permissions from permissions@acm.org.

DroneCom'20, September 25, 2020, London, United Kingdom

(C) 2020 Association for Computing Machinery.

ACM ISBN 978-1-4503-8105-5/20/09...\$15.00

https://doi.org/10.1145/3414045.3415946

\section{KEYWORDS}

D2D, Energy Efficiency, NOMA, Full Duplex, and WPCN.

ACM Reference Format:

Ishan Budhiraja, Neeraj Kumar, Mamoun Alazab, Sudhanshu Tyagi, Sudeep Tanwar, and Gaurav Srivastava. 2020. Energy Management Scheme for Wireless Powered D2D Users With NOMA Underlaying Full Duplex UAV. In Proceedings of DroneCom2020 . ACM, New York, NY, USA, 6 pages. https:// doi.org/10.1145/3414045.3415946

\section{INTRODUCTION}

D2D communications is a technique in which proximity devices communicate directly with each other with or without routing through the base station (BS). In this way, it offloads the data traffic and thus reduces the pressure from the BS. Also, D2D improves network spectral and energy efficiency (EE) by sharing the resources and supporting short distance communication [1]. Despite these advantages, D2D communications become more challenging when the infrastructure gets damaged. To address this challenge, researchers from both academia and industry proposed a new technology named as $\mathrm{UAV}[1]$.

UAV is a flying BS which is mainly used to set up the communication between the devices when the cellular infrastructure gets damaged. It acts as a static or mobile relay to extend the coverage and improve the quality-of-service (QoS) of the CEUEs. Also, by optimizing its trajectory, the communication links between devices are significantly improved which further results into higher throughput. Despite these advantages, it has been observed that both the UAV and D2D users are battery-constrained devices. It means they have a limited energy storage capacity which results in the degradation of an EE of the network. Hence, to prolong the lifetime of a battery, $\mathrm{EH}$ or wireless powered communication (WPC) have emerged as promising solutions [2]-[3].

The WPC is a harvest-then-transmit protocol and is a combination of the downlink wireless energy transfer (WET) and the uplink wireless information transfer (WIT) [2]. In the downlink WET, users devices harvest energy from the radio frequency $(\mathrm{RF})$ signals to charge their battery. On the other 
side, in WIT, the users' devices transmit information to the desired user using the harvested energy. However, both these processes have some limitations. In WET, the devices located far away from the BS harvest less RF energy due to their low signal power whereas, in WIT, these devices consume more energy during transmission. Also, in WIT, the users mostly used TDMA to transmit the information. However, in TDMA, each user used a specific time slot to transmit information, thus limiting the number of connections and reducing the data rate. To overcome these limitations and improve the fairness among the users, the power domain non-orthogonal multiple access (PD-NOMA) is proposed [4].

In PD-NOMA, the signals of different users are multiplexed at the transmitter's side using superposition coding. Then, the transmitter transmitted the multiplexed signal to the users through the same channel with different power levels. To decode the desired user's signal and reduce the intra-user interference, the successive interference cancellation technique is used at the receiver's side. In PD-NOMA, generally, a large amount of power is assigned to the far users and lesser to the near users [5]. The authors in [6] proposed an effective scheme to deploy the multiple UAVs for ground users under the condition that UAV transmits with its minimum power. The authors in [7] proposed the throughput maximization scheme under the mobile relaying system with respect to the power and trajectory constraints. The authors in [8] studied the energy-efficient scheme between the UAV and ground users with the condition that UAV wings consumed fixed energy. The coexistence of D2D in an underlay of UAV was first studied in [9]. The authors in [9] used one UAV to set up the communication between the D2D pair, and UAV travels from one-stop point to another to serve the other D2D pairs. The authors in [10] proposed the joint trajectory and precoding scheme for UAV-assisted NOMA networks. In the above-mentioned schemes, the authors did not consider the EE with respect to the limited battery power of the users. This motivates us to design this paper. In this paper, we integrate D2D, WPC, and NOMA at one platform to maximize the $\mathrm{EE}$ of the network.

\subsection{Contributions}

The main contributions of this paper are given as follows:

- An optimal value of power for DMG is estimated keeping the energy harvesting time constant.

- An optimal value for energy harvesting time is evaluated for DMG by fixing the power of the DDT and CT.

- A joint time and power allocation scheme is designed to maximize the EE of an overall network.

\subsection{Organization}

This paper is organized as follows: Section 2 describes the network model and the problem formulation. Section 3 elaborate the proposed scheme through which the formulated problem is solved. Section 4 evaluates the performance evaluation of the proposed scheme and compared with the existing NOMA

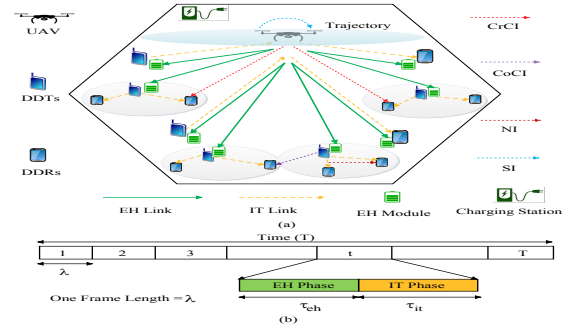

Figure 1: (a) System Model (b) Time Frame Structure.

\begin{tabular}{|c|c|c|}
\hline Sub-Frame I & Sub-Frame IIa & Sub-Frame IIb \\
\hline Time Duration $\left(\tau_{\mathrm{c}}\right)$ & Time Duration $\left(1-\tau_{\mathrm{e}}-\tau_{\mathrm{d}}\right)$ & Time Duration $\left(\tau_{\mathrm{d}}\right)$ \\
\hline NET from UAV-to-CT & $\begin{array}{l}\text { WIT from CT-to- } \\
\text { UAV }\end{array}$ & $\begin{array}{c}\text { WIT from UAV-to- } \\
\text { CR }\end{array}$ \\
\hline \multicolumn{3}{|c|}{ (a) Hop Mode } \\
\hline Sub-Frame I & \multicolumn{2}{|c|}{ Sub-Frame II } \\
\hline Time Duration $\left(\tau_{\mathrm{e}}\right)$ & \multicolumn{2}{|c|}{ Time Duration $\left(1-\tau_{\mathrm{c}}\right)$} \\
\hline $\begin{array}{l}\text { WET from UAV-to- } \\
\text { DDTs }\end{array}$ & \multicolumn{2}{|c|}{ IT from DDT-to-DDRs } \\
\hline
\end{tabular}

Figure 2: Harvest-Then-Transmit Model.

and TDMA schemes. Finally, Section 5 concludes the paper with the future scope.

\section{NETWORK MODEL}

\subsection{System Model with Assumptions}

Consider a single cell downlink scenario in which the UAV serve the set of $\mathcal{D}=\{1,2, \ldots, d, \ldots, D\}$ WP-DDGs, $\mathcal{I}=$ $\{1,2, \ldots, i, \ldots, I\} \mathrm{CT}$, and $\mathcal{J}=\{1,2, \ldots, j, \ldots, J\} \mathrm{CR}$ as shown in Fig. 1. Each WP-DDG consists of one DDT and two DDRs, $r_{1}$ and $r_{2}$. The number of DDRs in each DDG is restricted up to two for reduce the communication delay and hardware complexity. Firstly, in time $\tau_{e}$, the CTs, and the DDTs of each DDG harvest energy from the RF signals of the FD-UAV to store energy in its EH-module. Then, in time, $1-\tau_{e}-\tau_{d}$, the CT first transfer the information to FD-UAV, and then in time $\tau_{d}$, FD-UAV transfer the information to the $\mathrm{CR}$ to improve its data rate is shown in Fig. 2(a). On the other side, in time, $\tau_{h}=1-\tau_{e}$, the DDT used downlink PDNOMA to communicate with its respective DDRs as shown in Fig. 2(b). It should be noted that in the proposed model, we used the FD- UAV as a relay to reduce the transmission delay. Without loss of generality, assume a Cartesian system to set the coordinate of the UAV. Let us consider that the UAV flies at a fixed height $H$ from the ground within a finite time horizon $T$ as shown in Fig. 1(b). If total time $T$ is divided into $M$ slots, i.e., $\mathcal{M}=\{1,2, \ldots, m, \ldots, M\}$ with each having length $\lambda$, then the total time is represented as $T=\lambda M$. Then, the coordinate of the UAV during time slot $m$ is given as $\mathbb{U} m=x m, y m, H$. Also, assume that the start and end locations of the UAV are represented as $\mathbb{U}_{s} t=$ $x_{s} m, y_{s} m, H$ and $\mathbb{U}_{e} m=x_{e} m, y_{e} m, H$, respectively. If $v_{\max }$ is the maximum speed of the UAV, then the trajectory of the UAV at the $m^{t h}$ time frame holds the following inequality 
constraint:

$$
\|U m+1-U m\|^{2} \leq v_{\max } \lambda^{2}, \forall m \in \mathcal{M}
$$

The Cartesian coordinates of the $i^{\text {th }} \mathrm{CT}$ and the $j^{\text {th }} \mathrm{CR}$ are given as $\mathbb{U}_{i} m=x_{i} m, y_{i} m, 0$ and $\mathbb{U}_{j} m=x_{j} m, y_{j} m, 0$, respectively.

\subsection{Channel Model}

2.2.1 RM. When one CT wants to communicate with a $\mathrm{CR}$ via the relay (FD-UAV), then both the $\mathrm{CR}$ and FDUAV receive two different types of signals Non-Line-of-Sight (NLoS) and fading alongwith Line-of-Sight (LoS) signal. The aggregated path loss (PL) between the $i^{\text {th }} \mathrm{CT}$ to the FD-UAV alongwith LoS and NLoS signals is given as follows:

$$
\overline{P L}_{i, s}=P r_{i, s}^{L o S} P L_{i, s}^{L o S}+\left(1-P r_{i, s}^{N L o S}\right) P L_{i, s}^{N L o S} .
$$

where $P L_{i, s}^{L o S}=\Omega_{L O S}\left(\frac{4 \pi d_{i, s}}{\lambda_{0}}\right), P L_{i, s}^{N L o S}=\Omega_{N L O S}\left(\frac{4 \pi d_{i, s}}{\lambda_{0}}\right)$, $\operatorname{Pr}_{i, s}^{L o S} \tau=\frac{1}{1+a e^{-b \Theta_{i, s}-a}}$, and $\operatorname{Pr}_{i, s}^{N L o S}=1-\operatorname{Pr}_{i, s}^{L o S}$. Here $\lambda_{0}$ is the wavelength of the RF signal. $\Omega_{L o S}$ and $\Omega_{N L o S}$ are the LoS and NLoS signal loss arise due to the shadowing effect and fading. $d_{i, s}=\left\|\mathbb{U}_{s}-\mathbb{U}_{i}\right\|$ is the distance between the $i^{t h} \mathrm{CT}$ and the FD-UAV, $\Theta_{i, s}=180 \pi \sin ^{-1}\left(\frac{z_{s}}{d_{i, s}}\right)$ is an elevation angle between the $i^{\text {th }} \mathrm{CT}$ to the FD-UAV, and $a$ and $b$ are constant values. The channel gain between the $i^{\text {th }}$ CCUE to the UAV, and the UAV to the $j^{\text {th }}$ CEUE is given as follows:

$$
h_{i, s}=\frac{1}{\overline{P L}_{i, s}}, h_{s, j}=\frac{1}{\overline{P L}_{s, j}} .
$$

2.2.2 DM. In this mode, the $d^{\text {th }}$ DDT communicates directly with its two DDRs using NOMA. Therefore, the channel gain between the DDT and DDR $r_{1}$ and $r_{2}$ is represented as $h_{d, 1}$ and $h_{d, 2}$, respectively.

\subsection{UAV Consumption and Charging Model}

The power consumed by the UAV to serve the $j^{\text {th }} \mathrm{CR}$ is given as follows:

$$
P_{s}^{r}=\alpha_{s} \Sigma_{j=1}^{J} \phi_{s, j} P_{s, j}+\beta_{s}
$$

where $\alpha_{s}$ represents the internal consumption losses, $\beta_{s}$ is the offset losses, and $P_{s, j}$ is the transmission power from the UAV towards the $j^{\text {th }} \mathrm{CR}$.

The power consumed by the hovering and hardware of the UAV during EH and IT process is given as follows:

$$
P_{s}^{h d}=\sqrt{\frac{w g \rho}{2 \pi r_{p}^{2} N_{p}}}+\left(\frac{P^{m}-P^{s}}{v}\right) v_{s}+P^{s},
$$

where $w$ is the weight of the $\mathrm{UAV}, g$ is the gravity, and $\rho$ is the density, $r_{p}$ is the radius of the propeller, and $N_{p}$ is the number of the propeller. $P^{m}$ and $P^{s}$ are the power consumed by the UAV when it is moving with full speed, and at the static position, respectively. In static position, i.e., $v_{u}=0$.

Thus, the total power consumed by the UAV over $t$ is given as follows:

$$
P_{s}^{t}=P_{s}^{r}+P_{s}^{h d} \text {. }
$$

The energy consumed by the UAV during time $t$ from the wireless charger located at point $\mathbb{U}_{s}$ is given as follows:

$$
E_{s}^{c}=P_{u}^{t}\left(\frac{\left\|\mathbb{U} m-\mathbb{U}_{c}\right\|^{2}}{\left\|\mathbb{U} m-\mathbb{U}_{c}\right\|^{2}+\hat{\mathbb{U}}}\right),
$$

where $\widehat{W} \approx 0.0001$ is a very small positive number.

The energy used in charging the battery of the UAV over $t$ by the wireless charger is given as follows:

$$
E_{s}^{h}=\eta P_{c s}\left(1-\frac{\left\|\mathbb{U}_{s}-\mathbb{U}_{c}\right\|^{2}}{\left\|\mathbb{U}_{s}-\mathbb{U}_{c}\right\|^{2}+\hat{\mathbb{U}}}\right),
$$

where $\eta$ is the energy conversion efficiency, and $P_{c s}$ is the signal power from the charging station.

The energy stored in the UAV at the end of time slot $t$ from the wireless charger is given as follows:

$$
E_{s}^{s t r} t=E_{u}^{s t r}(t-1)+E_{u}^{h}-E_{u}^{c} .
$$

\subsection{WPCN Model}

This model is a combination of WET and WIT processes. In WET, the devices harvest the energy from the UAV, and in WIT, the devices transmit the data to receivers using the harvested energy. If the total time for one frame is $T$, then the time consumed for WET and WIT process is denoted as $\tau_{e}$ and $\tau_{d}$, respectively. Thus, the following equation holds:

$$
\tau_{e}+\tau_{d} \leq T \text {. }
$$

2.4.1 RM. In this mode, the CT communicates with the CR using the FD-UAV.

Case-I WET Process: The energy harvested by the $i^{\text {th }} \mathrm{CT}$ from the FD-UAV over $\tau_{e}$ is given as follows:

$$
E_{i}^{e h}=\eta \tau_{e} P_{s} h_{s, i}
$$

where $P_{s}$ is the transmit power of the UAV.

Case-II WIT Process: The signal transmitted by the $i^{\text {th }}$ $\mathrm{CT}$ to the FD-UAV over $1-\tau_{e}-\tau_{d}$ is given as follows:

$$
\begin{aligned}
y_{i, s}^{R M} & =\underbrace{\sqrt{P_{i} \psi_{i} h_{i, s}} x_{i, s}}_{\text {Desired Signal }}+\alpha_{i, s} \Sigma_{i^{\prime} \neq i}^{I} \underbrace{\sqrt{P_{i^{\prime}} \psi_{i} h_{i^{\prime}, s}} x_{i^{\prime}, s}}_{\text {Co-Channel (CCI) }} \\
& +\underbrace{\sqrt{P_{s} h_{s, s}} x_{s, s}}_{\text {Self Interference (SI) }}+\underbrace{\zeta_{i, s}}_{\text {AWGN }},
\end{aligned}
$$

Here, $\alpha_{i, s}$ is the resource block indicator. If $\alpha_{i, s}=1$, then the RB is allocated to the $i^{\text {th }} \mathrm{CT}$ to transmit an information to the FD-UAV, and otherwise $\alpha_{i, s}=0$, and $\psi_{i}$ represents the fraction of channel coefficient available for transmission from $i^{\text {th }} \mathrm{CT}$ to the FD-UAV.

The Signal-to-interference noise ratio (SINR) using (12) is calculated as follows:

$$
\Gamma_{i, s}^{R M}=\frac{P_{s} \psi_{i}\left|h_{i, s}\right|}{P_{i^{\prime}} \psi_{i} h_{i^{\prime}, s}+P_{s, s} h_{s, s}+\sigma^{2}} .
$$

The signal transmitted by the FD-UAV to the $j^{\text {th }} \mathrm{CR}$ is given as follows:

$$
y_{s, j}^{H M}=\sqrt{P_{s} \nu_{j} h_{s, j}} x_{s, j}+\Sigma_{d=1}^{D} \sqrt{P_{d} h_{d, j}} x_{d, j}+\zeta_{s, j},
$$


where $\nu_{j}$ denotes the fraction of channel coefficient available for transmission from the FD-UAV to the $j^{\text {th }} \mathrm{CR}$.

The SINR using (14) is calculated as follows:

$$
\Gamma_{s, j}^{R M}=\left(\frac{P_{s} \nu_{j}\left|h_{s, j}\right|}{\sum_{d=1}^{D} P_{d} h_{d, j}+\sigma^{2}}\right) .
$$

The data rate using (13) and (15) are calculated given as follows:

$$
\begin{gathered}
R_{i, s}^{R M}=\frac{B}{2}\left(1-\tau_{e}-\tau_{d}\right) \log _{2}\left(1+\Gamma_{i, s}^{R M}\right) . \\
R_{s, j}^{R M}=\frac{B}{2} \tau_{d} \log _{2}\left(1+\Gamma_{s, j}^{R M}\right) .
\end{gathered}
$$

where $\left(\frac{B}{2}\right)$ denotes the two hops of RM.

The data rate from the $i^{\text {th }} \mathrm{CT}$ to the $j^{\text {th }} \mathrm{CR}$ using FD$\mathrm{UAV}$ is defined as minimum of $R_{i, s}$ and $R_{s, j}$ which is given as follows:

$$
R_{i, j}^{R M}=\alpha_{i, j} \frac{B}{2} \min \left\{R_{i, u}^{R M}, R_{u, j}^{R M}\right\},
$$

where $\alpha_{i, j}=\left\{\alpha_{i, s}, \alpha_{a, j}\right\}$.

2.4.2 DM. In this mode, the $d^{\text {th }}$ communicates directly with the DDRs over time $1-\tau_{e}$.

Case-I WET Process: The energy harvested by the $d^{\text {th }}$ DDT from the FD-UAV over $\tau_{e}$ is given as follows:

$$
E_{d}^{e h}=\eta \tau_{e} P_{s} h_{s, d}
$$

Case-II WIT Process: The multiplexed signal transmitted by the $d^{t h}$ DDT to the $r_{1}$ DDR using downlink PD-NOMA is given as follows:

$$
\begin{aligned}
& y_{d, r_{1}}^{D M}=(\underbrace{\sqrt{P_{d} \theta_{d, r_{1}} h_{d, r_{1}}} x_{d, r_{1}}}_{\text {Desired Signal }}+\underbrace{\sqrt{P_{d} \theta_{d, r_{2}} h_{d, r_{2}}} x_{d, r_{2}}}_{\text {NOMA Interference } I_{d, r_{1}}^{n_{0}}})+\underbrace{\zeta_{d, r_{1}}}_{\text {AWGN }} \\
& +\underbrace{\sum_{d^{\prime} \neq d} \alpha_{d^{\prime}, d} \sqrt{P_{d^{\prime}} h_{d^{\prime}, r_{1}}} x_{d^{\prime}, r_{1}}}_{\text {Co-channel interference } I_{d^{\prime}, r_{1}}^{c o}}+\underbrace{\sqrt{P_{i} h_{i, r_{1}}} x_{i, r_{1}}}_{\text {Cross Channel interference } I_{i, r_{1}}^{c r}},
\end{aligned}
$$

where $P_{d}^{D M}=\left(\frac{\beta \eta \tau_{e} P_{s} g_{u, i}}{1-\tau_{e}}\right)$ and $P_{d^{\prime}}^{D M}$ are the transmit power of the $d^{\text {th }}$ and $d^{\prime t h}$ DDT, respectively. $\alpha_{d, r_{1}}$ and $\alpha_{d, r_{1}}$ are the power allocation factor assigned to $r_{1}$ and $r_{2}$ DDR. $x_{d, r_{1}}$ and $x_{d, r_{2}}$ are the signals between the $d^{\text {th }}$ DDT to the $r_{1}$ DDR, and that between the $d^{\text {th }}$ DDT to the $r_{2}$ DDR, respectively. $x_{d^{\prime}, r_{1}}$ is the co-channel interfering signal from the $d^{\prime t h}$ DDT to the $r_{1}$ DDR. $\alpha_{d^{\prime}, d}$ represents the co-channel interference, i.e., $\alpha_{d^{\prime}, d}=1$, then $d^{\prime t h}$ and $d^{\text {th }}$ DDT occupies the same RB, otherwise $\alpha_{d^{\prime}, d}=0$. Assume that $h_{d, r_{1}}>h_{d, r_{2}}$

Now, the SINR received at the $r_{1}$ DDR using (20) is given as follows:

$$
\Gamma_{d, r_{1}}^{D M}=\frac{\beta \eta \tau_{e} P_{s} h_{s, d} \alpha_{d, r_{1}} h_{d, r_{1}}}{I_{d, r_{1}}^{n o}+I_{d^{\prime}, r_{1}}^{c o}+I_{i, r_{1}}^{c r}+\sigma^{2} 1-\tau_{e}} .
$$

According to the principle of SIC, the SINR received at the $r_{2}$ DDR from the $d^{\text {th }}$ DDT is given as follows:

$$
\Gamma_{d, r_{2}}^{D M}=\frac{\beta \eta \tau_{e} P_{s} h_{s, i} \alpha_{d, r_{2}} h_{d, r_{2}}}{I_{d^{\prime}, r_{2}}^{c o}+I_{i, r_{2}}^{c r}+\sigma^{2} 1-\tau_{e}} .
$$

Now, the data rate of the $r_{1}$ and $r_{2}$ DDR is given as follows:

$$
\begin{aligned}
& R_{d, r_{1}}^{D M}=\left(1-\tau_{e}\right) B \log _{2}\left(1+\Gamma_{d, r_{1}}^{D M}\right) . \\
& R_{i, j_{2}}^{D M}=\left(1-\tau_{e}\right) B \log _{2}\left(1+\Gamma_{d, r_{2}}^{D M}\right) .
\end{aligned}
$$

The total data rate of the $d^{\text {th }}$ DMG is given as follows:

$$
R_{d}^{D M}=R_{d, r_{1}}^{D M}+R_{d, r_{2}}^{D M}
$$

\subsection{Energy Efficiency Model}

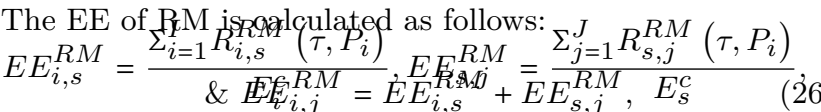
where $E_{i}^{c}=\tau_{e}+\tau_{d} P_{c}+\tau_{d} P_{i}$. Here, $\tau \in\left\{\tau_{e}, \tau_{d}\right\}, \alpha \in$ $\left\{\alpha^{R M}, \alpha^{D M}\right\}$, and $P_{c}$ is the power consumed by an internal circuits.

The EE of DM is calculated as follows:

$$
E E_{d}^{D M}=\frac{\sum_{d=1}^{D} R_{d}^{D M}(\tau, \theta)}{E_{d}^{c}} .
$$

where $E_{d}^{c}=\tau_{e}+\tau_{d} P_{c}+\tau_{d} P_{d}$, and $\theta \in\left\{\theta_{d, r_{1}}, \theta_{d, r_{2}}\right\}$.

\subsection{Problem Formulation}

In this paper, our goal is to maximize the EE of an overall network with respect to the time and power. The mathematical formulation of the problem is described as follows:

$$
\begin{aligned}
& \text { P.F. : } \max _{\tau, \theta} E E_{d}^{D M} \text {, } \\
& \text { s.t. } C_{1} \quad: \quad \tau_{e}+\tau_{d} \leq T, C_{2}: \tau_{e}, \tau_{d} \geq 0 \text {, } \\
& C_{3}: R_{d, r_{1}} \geq R_{d, r_{1}}^{\min }, R_{d, r_{2}} \geq R_{d, r_{2}}^{\min }, \forall j \in \mathcal{J}, d \in \mathcal{D} \\
& C_{4}: E_{d}^{c} \leq E_{d}^{e h}, C_{5}: \frac{\left\|\mathbb{U}_{s} m+1-\mathbb{U}_{s} m\right\|^{2}}{v} P_{s}^{h d}+E_{s}^{c} \leq E_{s} \text {, } \\
& C_{6}: \theta_{d, r_{1}}+\theta_{d, r_{2}} \leq 1, C_{7}: \theta_{d, r_{1}}, \theta_{d, r_{2}} \geq 0, \forall d \in \mathcal{D} \text {. }
\end{aligned}
$$

In the above-mentioned problem, constraint $C_{1}$ ensures that the time consumed in WET and WIT process in one time frame must be less than $T$. Constraint $C_{2}$ represents the non-negative WET and WIT time. Constraint $C_{3}$ implies the minimum data rate requirements of the $r_{1}$ and $r_{2}$ DDRs. $C_{4}$ ensures that the energy consumed by the $d^{\text {th }}$ DDT should be less than the harvested energy. Constraint $C_{5}$ implies that UAV must retain an energy less than the stored energy in its battery to reach the charging station. Constraint $C_{6}$ ensures the upper limit of the transmission power of the DDTs, and $C_{7}$ ensures the non-negative power transmission.

\section{PROPOSED SCHEME}

In this section, we propose an algorithm for problem (28) to maximize the EE by jointly optimizing the energy harvesting time and power allocation. To solve the problem (28), we first change the variables [9].

$\tau_{d}=1-\tau_{e}=\frac{1}{\lambda}, P_{d} \alpha_{d}=\frac{1}{\alpha_{d} P_{d}}, \forall d \in \mathcal{D}$ and $P_{i}=\frac{1}{P_{i}}, \forall i \in \mathcal{I}$.

such that the variable satisfy the convex constraint

$$
\lambda>1
$$


Now, the problem in (28) is rewritten as follows:

$$
\begin{array}{ll} 
& \text { P.F. }: \max _{\tau, \theta} E E_{d}^{D M}, \\
\text { s.t. } \quad: \lambda>1, \frac{1}{P_{d}} \leq(\lambda-1) \eta P_{d} h_{s, d}, \\
\quad: \frac{1}{\lambda} \log _{2}\left(1+\frac{h_{d, r_{1}}}{P_{d} \theta_{d, r_{1}} I_{d, r_{1}}^{n o}+I_{d^{\prime}, r_{1}}^{c o}+I_{i, r_{1}}^{c r}+\sigma^{2}}\right), \\
& : \frac{1}{\lambda} \log _{2}\left(1+\frac{h_{d, r_{2}}}{P_{d} \theta_{d, r_{2}} I_{d^{\prime}, r_{2}}^{c o}+I_{i, r_{2}}^{c r}+\sigma^{2}}\right), C_{5}-C_{7}
\end{array}
$$

where $\Sigma_{i=1}^{I} \frac{1}{\lambda P_{i}}+1-\frac{1}{\lambda} \eta P_{s}+P_{c}$ and $\Sigma_{d=1}^{D} \frac{1}{\lambda P_{d}}+1-\frac{1}{\lambda} \eta P_{s}+P_{c}$.

To solve the problem (31), we use the logarithmic inequality

$$
\begin{aligned}
& \frac{1}{\rho} \log _{2} 1+\frac{1}{u v} \geq \frac{2}{\bar{\rho}} \log _{2} 1+\frac{1}{\bar{u} \bar{v}}+\frac{2}{\bar{\rho} \bar{u} \bar{v}+1} \\
& -\frac{1}{\bar{\rho} \bar{u} \bar{u} \bar{v}+1} u-\frac{1}{\bar{\rho} \bar{v} \bar{u} \bar{v}+1} v-\frac{\log _{2} 1+\frac{1}{\bar{u} \bar{v}}}{\bar{\rho}^{2}} \rho
\end{aligned}
$$

which follows from the convexity of function $\log _{2} 1+\frac{1}{u v} \rho$.

For $u=\frac{\theta P_{d}}{h_{d, r_{1}}}, v=\frac{h_{d, r_{1}}}{\theta P_{d}}+\Sigma_{d^{\prime} \neq d}^{D} \frac{h_{d^{\prime}, r_{1}}}{P_{d}}+\frac{h_{i, r_{1}}}{P_{i}}+\sigma^{2}, \rho=\lambda$, $\bar{u}=u^{\iota}=\frac{\theta P_{d}^{\iota}}{h_{d, r_{1}}}, \bar{v}=v^{\iota}=\frac{h_{d, r_{1}}}{\theta P_{d}^{\iota}}+\sum_{d^{\prime} \neq d}^{D} \frac{h_{d^{\prime}, r_{1}}}{P_{d}^{\iota}}+\frac{h_{i, r_{1}}}{P_{i}^{\iota}}+\sigma^{2}$, $\bar{\rho}=\rho^{\iota}=\lambda^{\iota}$.

Now, the sum rate is rewritten as follows:

$$
R_{d,} \lambda, P \geq \varphi_{d, r_{1}}^{\iota} \lambda, P
$$

where $\varphi_{d}^{\iota} \lambda, P=\frac{2}{\rho^{\iota}} \log _{2} 1+\frac{1}{u^{\iota} v^{\iota}}+\frac{2}{\rho^{\iota} u^{\iota} v^{\iota}+1}$

$$
-\frac{1}{\rho^{\iota} u^{\iota} u^{\iota} v^{\iota}+1} u-\frac{1}{\rho^{\iota} v^{\iota} u^{\iota} v^{\iota}+1} v-\frac{\log _{2} 1+\frac{1}{u^{\iota} v^{\iota}}}{\rho^{\iota}} \rho
$$

With the feasible points $\lambda^{\iota}, P^{\iota}$ of (31), we have

$$
\delta^{\iota}=\Sigma_{d=1}^{D} \frac{\varphi_{d, r_{1}}^{\iota} \lambda^{\iota}, P^{\iota}}{\Psi \lambda^{\iota}, P^{\iota}}
$$

where $\Psi \lambda^{\iota}, P^{\iota}=E_{d}^{c}$

At the $\iota^{\text {th }}$ iteration, the following convex program is solved to generate the next feasible point.

$$
\max _{\lambda, P} \Sigma_{d=1}^{D} \varphi_{d, r_{1}}^{\iota} \lambda^{\iota}, P^{\iota}-\delta^{\iota} \Psi \lambda^{\iota}, P^{\iota}
$$

We propose an algorithm to solve the EE maximization (36). The initial point $\lambda^{0}, P^{0}$ for (36) is easily located by random search such that it satisfies the constraints in problem (31).

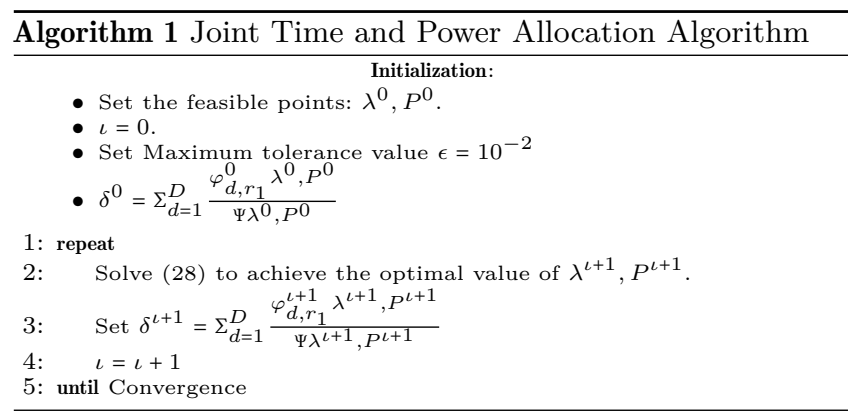

\subsection{Power Allocation}

This algorithm addresses power allocation for EE maximization problem (28) where the harvesting time value is fixed as $1-\tau_{e}=\frac{1}{\lambda^{*}}, \lambda^{*}>1$. Now, the equation (28) is rewritten as follows:

$$
\begin{array}{ll}
\text { P.F. } & : \quad \max _{P} E E_{d}^{D M} \\
\text { s.t. } & \theta_{d} P_{d} \leq \lambda^{*}-1 \eta P_{s} h_{s, d} \\
& R_{d, r_{1}} \geq \lambda^{*} R_{d, r_{1}}^{\min }, R_{d, r_{2}} \geq \lambda^{*} R_{d, r_{2}}^{\min },
\end{array}
$$

To solve the non-convex problem (37), we used the inequality derived in (32).

Let $u=\frac{1}{\theta P_{d} h_{d, r_{1}}}, v=\theta P_{d} h_{d, r_{2}}+\sum_{d^{\prime} \neq d^{\prime}}^{D} P_{d^{\prime}} h_{d^{\prime}, r_{1}}+P_{i} h_{i, r_{1}}+$ $\sigma^{2}, \rho=1$, and $\bar{u}=u^{\iota}=\frac{1}{\theta P_{d}^{\iota} h_{d, r_{1}}}, \bar{v}=v^{\iota}=\theta P_{d}^{\iota} h_{d, r_{2}}+$ $\sum_{d^{\prime} \neq d^{\prime}}^{D} P_{d^{\prime}}^{\iota} h_{d^{\prime}, r_{1}}+P_{i}^{\iota} h_{i, r_{1}}+\sigma^{2} \bar{\rho}=\rho^{\iota}=1$.

Then, the numerator of objective function in (37) is rewritten as follows:

$$
R_{d}^{D M} \lambda^{*}, P \geq \bar{\varphi}_{d}^{\iota} \lambda^{*}, P
$$

At the $\iota^{\text {th }}$ iteration, the following convex program is solved to generate the next feasible point.

$$
\begin{array}{ll} 
& \max _{\lambda, P} \Sigma_{d=1}^{D} \varphi_{d, r_{1}}^{\iota} \lambda^{\iota}, P^{\iota}-\delta^{\iota} \Psi \lambda^{\iota}, P^{\iota} \\
\text { s.t. } & \theta_{d} P_{d} \leq \lambda^{*}-1 \eta P_{s} h_{s, d} \\
& R_{d, r_{1}} \geq \lambda^{*} R_{d, r_{1}}^{\min }, R_{d, r_{2}} \geq \lambda^{*} R_{d, r_{2}}^{\min }, C_{6}-C_{7}
\end{array}
$$

\subsection{Time Allocation}

This algorithm solves the harvesting time optimization problem in the slack variable of $\lambda$ with the use of maximum harvested power in $\mathrm{D} 2 \mathrm{D}$ communication as follows:

$$
P_{d}=(\lambda-1) \eta P_{s} h_{s, d}
$$

Therefore, the max-min sum-rate problem with fixed harvested energy is given as follows:

$$
\begin{gathered}
\max _{\lambda} \min _{d \in \mathcal{D}} \frac{1}{\lambda} \log _{2} \\
\left(1+\frac{(\lambda-1) h_{d, r_{1}} g_{r_{1}}}{\lambda-1 h_{d, r_{2}} g_{r_{2}}+\sum_{d^{\prime} \neq d}^{D} h_{d, r_{1} g_{r_{1}}}+h_{i, r_{1} g_{r_{1}}+\sigma^{2}}}\right)
\end{gathered}
$$

Next, the objective function in (41) can be approximated by using the inequality (32) for $u=\frac{1}{(\lambda-1)}$ Let $u=\frac{1}{(\lambda-1)} h_{d, r_{1}}$, $v=(\lambda-1) P_{d} h_{d, r_{2}}+\Sigma_{d^{\prime} \neq d}^{D} P_{d^{\prime}} h_{d^{\prime}, r_{1}}+P_{i} h_{i, r_{1}}+\sigma^{2}, \rho=1$, and $\bar{u}=u^{\iota}=\frac{1}{\theta P_{d}^{\iota} h_{d, r_{1}}}, \bar{v}=v^{\iota}=\theta P_{d}^{\iota} h_{d, r_{2}}+\sum_{d^{\prime} \neq d^{\prime}}^{D} P_{d^{\prime}}^{\iota} h_{d^{\prime}, r_{1}}+$ $P_{i}^{\iota} h_{i, r_{1}}+\sigma^{2} \bar{\rho}=\rho^{\iota}=1$.

Then, the numerator of objective function in (37) is rewritten as follows:

$$
R_{d}^{D M} \lambda^{*}, P \geq \bar{\varphi}_{d}^{\iota} \lambda^{*}, P
$$

At the $\iota^{\text {th }}$ iteration, the following convex program is solved to generate the next feasible point. 

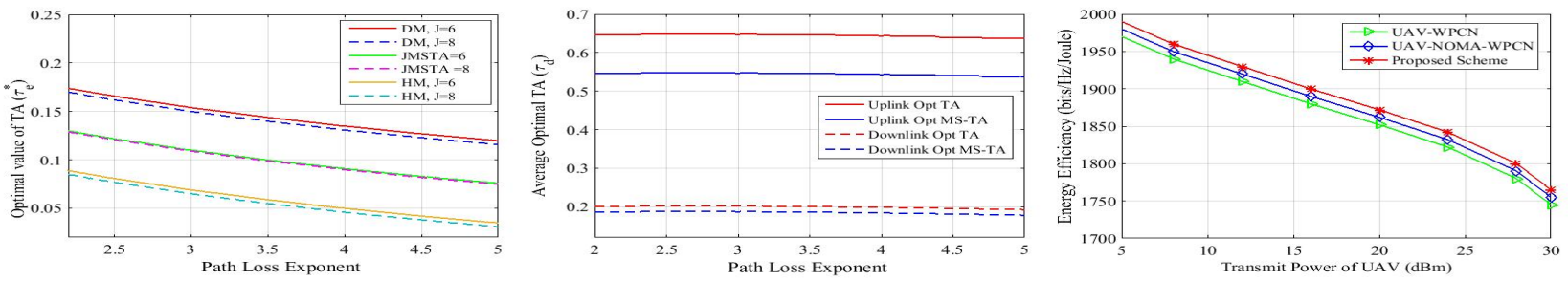

Figure 3: Comparative Analysis: (a) Optimal Value of TA for DM v/s Path loss exponent (b) Optimal Value of TA for DM v/s Path loss exponent (c) Energy Efficieny of network v/s Transmit power of UAV.

\section{PERFORMANCE EVALUATION}

In this section, performance of the proposed scheme is evaluated. The simulation parameters used in the evaluation are taken from [2] as shown in Table 1 . The UAV and charging station is located at coordinates $(400,400,60)$ and $(200,200,60)$, respectively.

Fig. 3(a) describes the variation in optimal value of TA Table 1: Simulation Parameters

\begin{tabular}{|ll|ll|}
\hline Parameters & Values & Parameters & Values \\
\hline Cell Layout & Hexagonal & $a, b$ & $9.6,2.9$ \\
Cell Radius & $60 \mathrm{~m}$ & Bandwidth $B$ & $20 \mathrm{MHz}$ \\
Area & $800 \mathrm{~m} \times 800 \mathrm{~m}$ & $z$ (elevation) & $60 \mathrm{~m}$ \\
$\lambda_{0}$ & $0.125 \mathrm{~m}$ & $\Omega_{L O S}$ & 1 \\
$\Omega_{N L O S}, \mathcal{I}$ & 12,10 & $\alpha_{u}, N_{p}$ & 4,5 \\
$\beta_{u}$ & 6.8 & $P_{c s}$ & $10 \mathrm{~W}$ \\
$P_{u}^{\text {max }}$ & $25 \mathrm{dBm}$ & $P_{i}^{\text {max }}$ & $25 \mathrm{dBm}$ \\
$w, r_{p}$ & $1 \mathrm{Kg}, 20 \mathrm{~cm}$ & $P^{s}$ & $0.5 \mathrm{~W}$ \\
$v, \iota_{\text {max }}$ & $15 \mathrm{~m} / \mathrm{s}, 15$ & $E_{i}^{e h}$ & $15 \mathrm{KJ}$ \\
\hline for DM with respect to the path loss exponent. The result
\end{tabular}

shows that the proposed JMSTA achieves the optimal TA in between the DM and HM. This happened because the link quality in DM is much better than that in HM due to short distance between the DDT and DDRs in each DMG.

Fig. 3(b) shows the graph between the optimal value of TA for DM with respect to the path loss exponent. The results in graph implies that the proposed scheme in both the uplink and downlink scenarios achieve $1.34 \%$ and $0.1 \%$ approximately equals to the optimal value.

Fig. 3(c) shows the impact on the EE of a network with respect to the transmit power of UAV. The results shows that when the transmit power of UAV increases, then the proposed scheme achieves higher EE as compared to the pre-existing NOMA [2] and OMA [8] scheme. This happened because with an increases in transmit power of UAV, the DDT users harvest large amount of energy from the UAV and easily transmit the information to DDRs, resulting into an increase in EE of the DMG.

\section{CONCLUSION}

In this paper, we have proposed the energy efficient resource allocation scheme for wireless powered D2D Users with NOMA underlaying FD-UAV. Here, the DDTs first harvest energy from the RF signal of the FD-UAV, and then communicate with the DDRs using NOMA technique. The formulated problem is in a MINLP form, so to solve this we divide it into two sub-problems. In the first sub-problem, an optimal value of power is estimated, whereas in the second sub-problem, an optimal vale of energy harvesting time is calculated using the concept logarithmic equality. Finally, a joint time and power allocation algorithm is designed to maximize the EE of the DMGs. In future, we will extend this scheme to a multiple UAV scenario.

\section{ACKNOWLEDGEMENT}

This work was supported by the Department of Corporate and Information Services, NTG of Australia.

\section{REFERENCES}

[1] Q.-V. Pham, F. Fang, V. N. Ha, M. Le, Z. Ding, L. B. Le, and W.-J. Hwang, "A Survey of Multi-Access Edge Computing in 5G and Beyond: Fundamentals, Technology Integration, and State-ofthe-Art," arXiv preprint arXiv:1906.08452, Jun. 2019.

[2] J. Xu, Y. Zeng, and R. Zhang, "UAV-Enabled Wireless Power Transfer: Trajectory Design and Energy Optimization," IEEE Transactions on Wireless Communications, vol. 17, no. 8, pp. 5092-5106, Aug. 2018.

[3] J. Yang, S. Xiao, B. Jiang, H. Song, S. Khan, and S. Ul Islam, "Cache-enabled unmanned aerial vehicles for cooperative cognitive radio networks," IEEE Wireless Communications, vol. 27, no. 2, pp. 155-161, Mar. 2020.

[4] I. Budhiraja, S. Tyagi, S. Tanwar, N. Kumar, and J. J. P. C. Rodrigues, "DIYA: Tactile Internet Driven Delay Assessment NOMA-Based Scheme for D2D Communication," IEEE Transactions on Industrial Informatics, vol. 15, no. 12, pp. 6354-6366, Dec. 2019.

[5] I. Budhiraja, S. Tyagi, S. Tanwar, N. Kumar, and J. J. P. C. Rodrigues, "Tactile Internet for Smart Communities in 5G: An Insight for NOMA-Based Solutions," vol. 15, no. 5, May 2019, pp. 3104-3112.

[6] M. Mozaffari, W. Saad, M. Bennis, and M. Debbah, "Optimal Transport Theory for Power-Efficient Deployment of Unmanned Aerial Vehicles," in IEEE international conference on communications (ICC), Kuala Lumpur, Malaysia,, pp. 1-6, May 2016.

[7] Y. Zeng, R. Zhang, and T. J. Lim, "Throughput Maximization for UAV-enabled Mobile Relaying Systems," IEEE Transactions on Communications, vol. 64, no. 12, pp. 4983-4996, Sept. 2016.

[8] Y. Zeng and R. Zhang, "Energy-Efficient UAV Communication with Trajectory Optimization," IEEE Transactions on Wireless Communications, vol. 16, no. 6, pp. 3747-3760, Jun. 2017.

[9] M. Mozaffari, W. Saad, M. Bennis, and M. Debbah, "Unmanned Aerial Vehicle with Underlaid Device-to-Device Communications: Performance and Tradeoffs," IEEE Transactions on Wireless Communications, vol. 15, no. 6, pp. 3949-3963, Feb. 2016.

[10] N. Zhao, X. Pang, Z. Li, Y. Chen, F. Li, Z. Ding, and M.-S. Alouini, "Joint Trajectory and Precoding Optimization for UAVAssisted NOMA Networks," IEEE Transactions on Communications, vol. 67, no. 5, pp. 3723-3735, Jan. 2019. 